the associations among FADD (Fadd) expression, TIL proportion and patient prognosis or tumor development were evaluated by NanoString/immunohistochemistry analysis in HCC patients or RT-qPCR/flow cytometry in orthotopic HCC mouse models.

Results $\mathrm{CD}^{+} \mathrm{T}$ cell migration rate was significantly enhanced in FADD-oe tumors, but reduced in FADD-kd tumors in both HCC cells-T cell co-culture system in vitro and subcutaneous tumor models in vivo. Mechanistically, FADD activated NF- $\kappa$ $\mathrm{B}$, which in turn promoted $\mathrm{T}$ cell trafficking chemokine CCL5 production. Furthermore, Fadd expression was positively correlated with $\mathrm{Ccl} 5$ and TIL proportions, but negatively correlated with orthotopic HCC tumor weights. As we showed that FADD was positively correlated with $\mathrm{CD}^{+} \mathrm{T}$ cells in HCC patients, our data pinpointed that FADD controls TIL abundance in HCC.

Conclusions Our findings reveal an underlying mechanism of TIL accumulation in HCC, which provides a novel strategy of FADD activation in turning immunologically cold tumors into hot ones for better prognosis and immunotherapeutic responses.

\section{IDDF2021-ABS-0177 RNF135 PROMOTER METHYLATION IS ASSOCIATED WITH IMMUNE INFILTRATION AND PROGNOSIS IN HEPATOCELLULAR CARCINOMA}

Guijun Zhao*, Xiong Liang, Yu Bai, Hao Li, Guang Yong, Yusha Liu, Yukun Liang, Xiao Wang. Endoscopy Center, Inner Mongolia key laboratory of endoscopic digestive diseases, Inner Mongolia People's Hospital, Hohhot, Inner Mongolia Autonomous Region, China

\subsection{6/gutjnl-2021-IDDF.8}

Background RING finger protein 135 (RNF135) has an important role in the occurrence of many cancers, however, the regulation and function of RNF135 in hepatocellular carcinoma (HCC) remains unknown.

Methods The promoter methylation status and mRNA expression of RNF135 were evaluated by methylation-specific PCR and real-time PCR in HCC cell lines and tissues, and further analyzed from The Cancer Genome Atlas database. Transwell migration, wound healing assay, cell viability, and colony formation assay were performed to investigate the function of RNF135. GSEA analysis, TIMER database and ESTIMATE algorithm were used to decipher the associated pathway and immune infiltration. The survival analysis was applied to assess the prognostic value of RNF135.

Results RNF135 expression was downregulated in 5 of 8 HCC cell lines and HCC tissues, and was negatively correlated with its promoter hypermethylation. Demethylating regent decitabine restored RNF135 expression on the cellular level. Knockdown of RNF135 expression enhanced the migration of HCC cells, while RNF135 overexpression repressed cell migration. Bioinformatics analysis revealed a positive relationship between RNF135 expression and six immune cell infiltrates (B cells, CD4 + T cells, CD8 + T cells, neutrophils, macrophages, and dendritic cells). Survival analysis disclosed that RNF135 hypermethylation is independently associated with poor clinical outcomes in HCC.

Conclusions Decreased RNF135 expression driven by promoter hypermethylation is frequently occurred in HCC and associated with prognosis of HCC. RNF135 functions as a tumor suppressor and involves in tumor immune microenvironment in HCC.

\section{IDDF2021-ABS-0185 OVERCOME PRIMARY RESISTANCE TO IMMUNE CHECKPOINT BLOCKADE IN HEPATOCELLULAR CARCINOMA}

${ }^{1}$ Xiaoyu Liu*, ${ }^{1}$ Jingying Zhou, ${ }^{2}$ Man Liu, ${ }^{3}$ Ying Wang, ${ }^{3}$ Mengying Hu, ${ }^{3}$ Rihe Liu, ${ }^{3}$ Leaf Huang, ${ }^{1}$ Hanzhuang Liu, ${ }^{1}$ Alfred Sze-Lok Cheng. ' School of Biomedical Sciences, The Chinese University of Hong Kong, Hong Kong, China; ${ }^{2}$ The First Affiliated Hospital, Sun YatSen University, Guangzhou, China; ${ }^{3}$ Department of Biomedical Engineering, The Chinese University of Hong Kong, Hong Kong, China; ${ }^{4}$ Eshelman School of Pharmacy, University of North Carolina, Chapel Hill, NC, USA; ${ }^{5}$ Lee Kong Chian School of Medicine, Nanyang Technological University, Singapore; ${ }^{6}$ Institute of Precision Medicine, The First Affiliated Hospital, Sun Yat-sen University, Guangzhou, China

\subsection{6/gutjnl-2021-IDDF.9}

Background Although immune checkpoint blockade (ICB) such as anti-programmed cell death 1 or its ligand (anti-PD-1/PDL1) has revolutionized the treatment paradigm for cancer patients, most hepatocellular carcinoma (HCC) patients did not show benefits in the clinical scenario. The aim of this study is to investigate the underlying mechanisms of immunotherapy resistance in HCC and explore therapeutic approaches for enhancing HCC immunotherapy.

Methods Fibrosis-associated HCC mouse model was established by chemical induction and tumor inoculation. Non-alcoholic fatty liver disease (NAFLD)-associated HCC was established by high-fat high carbohydrate diet and tumor implantation. Integrated analysis of single-cell RNA sequencing and bulk RNA sequencing were applied to identify monocytic myeloid-derived suppressor cells (M-MDSC) signature genes. Immune profiles were determined by flow cytometry.

Results We found M-MDSC was specifically enriched in nonresponders and a protein phosphatase was identified as a novel signature gene in M-MDSC. Inhibition of this protein phosphatase suppressed M-MDSC expansion and reduced reactive oxygen species generation, which holds promise in ameliorating the immunosuppressive microenvironment for tumor eradication. To further improve HCC therapy in ICB manner, we found an elegant nanoparticle-parceled PD-L1 trap which was superior to the conventional antibody-mediated ICB therapy. This PD-L1 trap was more up taken by the fibrotic liver environment compared to the normal liver indicating it would be suitable for majority of HCC patients. Ultimately, the PDL1 trap triggered robust anti-tumor response in both fibrotic HCC and NAFLD-associated HCC. Mechanistically, PD-L1 trap exerted potent immune-editing function by diminishing immunosuppressive cells and boosting $\mathrm{CD} 8^{+} \mathrm{T}$ cells infiltration to the tumor. Those tumor-infiltrating $\mathrm{CD}^{+} \mathrm{T}$ cells were more cytotoxic with augmented cytokines production. More importantly, improved survival was observed in PD-L1 traptreated HCC.

Conclusions We uncovered a novel target for immunosuppressive myeloid cells which was closely associated with immunotherapy resistance in HCC. Moreover, a novel ICB approach showed translational potential for treating HCC with the enhanced therapeutic effect but reduced toxicity. We anticipate providing more efficacious options for HCC patients with long-term benefits. Acknowledgements: This study is supported by HMRF (16170451, 07180556) and $\mathrm{Li} \mathrm{Ka}$ Shing Foundation. 